\title{
Insomnia Treatment
}

\author{
Japheth Jaoko, Ph.D. \\ Carver School of Social Work and Counseling, Campbellsville University \\ 1 University Drive, UPO 855 \\ Campbellsville, KY 42718-2799 \\ E-mail: jojaoko@campbellsville.edu
}

Doi:10.5296/jsr.v5i1.5327 URL: http://dx.doi.org/10.5296/jsr.v5i1.5327

\begin{abstract}
This paper set out to investigate the effectiveness of insomnia treatment. Insomnia is defined as the inability to fall asleep or stay asleep. An online search was conducted to locate relevant empirical articles in three online databases - JSTOR, PsychInfo, and Academic Search Premier. The search was conducted using the following key words - 'sleep disorders', 'insomnia', 'sleep difficulties', and 'sleep disturbance'. Additional information was obtained from academic texts.

The paper begins with introductory matters about insomnia followed by the literature review. Various treatment approaches of insomnia are presented, followed by a critical analysis and social work implications.
\end{abstract}

Key Words: Sleep Disorders, Insomnia, Sleep Disturbance, Sleep Difficulties

\section{Introduction}

According to the American Psychiatric Association (2000), sleep disorders refers to a group of conditions that results in the disturbance of the normal sleep patterns, and that interferes with optimal functioning during the day. These conditions include insomnia, hypersomnia, narcolepsy, nightmares, and sleep disruptive behaviors such as sleepwalking. The focus of this paper will be limited to insomnia. Specifically, the paper will focus on the treatment of insomnia.

The term "insomnia" has its roots in Latin. 'In' means no, and 'sommus' means sleep, which when translated to English means inability to sleep or a total lack of sleep (Estivill et al., 2003). Insomnia is thus defined as the inability to fall asleep or stay asleep. The condition also includes early awakenings with the inability to fall asleep again. As a consequence, insomnia interferes with an individual's ability to get the right amount or quality of sleep necessary for optimal functioning (Monti, 2004). The amount of sleep required by individuals varies from person to person and is often dependent on the different stages of human development. On average however, teenagers need about nine hours of sleep while adults need about eight hours of sleep for optimal functioning.

\section{Literature Review}

Reliable prevalence data on insomnia in the United States is scarce. This is mainly 
because different scientific studies utilize different definitions and methods to explore the problem. According to Holcomb (2007), insomnia affects approximately 50 to 70 million Americans. Other studies report a prevalence rate of 5\% to 35\%, which roughly translates to 15 to 105 million Americans (Centers for Disease Control and Prevention, 2013; Drake, Roehrs, \& Roth, 2003; Estivill et al., 2003; Sateia, \& Nowell, 2004).

Insomnia is more common among older Americans. About $50 \%$ of individuals aged 65 and over in the United States experience insomnia (Lopez, 2013, \& Estivill et al., 2003). Insomnia is also common among women, individuals who are separated or divorced, medically ill patients, and those with depression, anxiety, or substance abuse issues (American Family Physician, 2005; Monti, 2004; Sateia, \& Nowell, 2004).

Insomnia can be classified as acute or chronic. Acute insomnia is short-term and usually last less than four weeks (Holcomb, 2007). About 10\% of those experiencing insomnia experience chronic insomnia (Monti, 2004). Chronic insomnia is long-term and involves sleeplessness for at least three or more nights each week for a period of more than one month (Holcomb, 2007). Chronic insomnia has been shown to last on average for four years, with many individuals continuing to suffer for ten or more years (Drake, Roehrs, \& Roth, 2003).

Insomnia can also be classified as mild, moderate, or severe (Estivill et al., 2003). Mild insomnia involves minimum impairment of functioning. Moderate insomnia involves moderate impairment of functioning and includes symptoms such as irritability, anxiety, and fatigue. Severe insomnia is associated with more critical symptoms such as depression (Estivill et al., 2003).

Insomnia has been shown to have a negative impact on individuals overall quality of life (Drake, Roehrs, \& Roth, 2003). Insomnia can lead to impaired judgment and coordination, resulting in a decline in an individual's driving ability, work or school performance, and other important areas of daily functioning (Drake, Roehrs, \& Roth, 2003; Holcomb, 2007). Insomnia can also to lead to mood swings, personality changes, fatigue, and even hallucinations (Holcomb, 2007). Additionally, the financial cost of insomnia related to lost productivity, work related accidents, absenteeism, and health care costs are enormous (Drake, Roehrs, \& Roth, 2003).

\section{Theories}

Theoretical models about insomnia include the diathesis-stress model and the light sleeper model. The diathesis-stress model postulates that a precipitating event in the environment interacts with an individual's biological or genetic predisposition to trigger insomnia. The light sleeper model postulates that individuals who suffer from insomnia are by nature "light" sleepers and thus may be more sensitive to sleep-disturbance stimuli in the environment (Drake, Roehrs, \& Roth, 2003).

In terms of treatment guide, it seems that the bio-psycho-social model is the best approach to use. This approach postulates that biological, psychological, and social factors all need to be taken into consideration when treating insomnia.

\section{Treatment of Insomnia}

Insomnia is a complex condition with many causal factors. These include stress, circadian rhythm issues, poor bedtime habits, illness, diet, medications, drugs and alcohol use, 
and the sleep environment (Drake, Roehrs, \& Roth, 2003; Estivill et al., 2003; Holcomb, 2007; Monti, 2004; Sateia \& Nowell, 2004). Likewise, there are a number of treatments programs available for those suffering from insomnia. These programs may include pharmacological approaches, behavioral approaches, or a combination of the two approaches.

\subsection{Pharmacological Approaches}

The Food and Drug Administration (FDA) approve two classes of drugs for the treatment of insomnia: benzodiazepines and melatonin-receptor agonists. Medications with short acting agents are used for sleep-onset insomnia whereas those with intermediate-acting or long-acting agents are used for sleep maintenance (Passarella, \& Duong, 2008).

Benzodiazepines such as prosom, dalmane, restoril, doral, and halcion are commonly prescribed for sleep insomnia (Passarella, \& Duong, 2008). Studies have shown that benzodiazepines increases total sleep time but has no significant effect on sleep latency. Side effects of benzodiazepines include daytime sleepiness, hangover effects, dizziness, and impaired memory (Winkelman, \& Pies, 2005). Furthermore patients taking benzodiazepines even for just a few days may develop dependence to the drug and thus patients may suffer withdrawal symptoms upon discontinuation of use (Passarella, \& Duong, 2008).

Ramelteon (rozerem) is a drug in a class of drugs known as melatonin-receptor agonists. Ramelteon is primarily used for the treatment of insomnias characterized by delayed sleep onset although evidence indicates that it also increases total sleep time (Passarella, \& Duong, 2008). Common side effects associated with ramelteon include fatigue and dizziness (Winkelman, \& Pies, 2005).

Some antidepressants such as trazodone and mirtazapine are also frequently prescribed for insomnia. Studies have shown trazodone to be effective in improving sleep latency and maintenance compared to placebo (Passarella, \& Duong, 2008). Side effects of trazodone include dizziness, sedation, hypotension, headache, and priapism. Antidepressants are typically more beneficial in patients with comorbid depression (Passarella, \& Duong, 2008).

Other non prescription drugs such as antihistamines and herbal products such as melatonin and valerian are also frequently used for insomnia. There is little evidence to support the efficacy of antihistamines (Passarella, \& Duong, 2008). Additionally, herbal products are not regulated by the FDA and their efficacy and safety have not been demonstrated.

Although certain sleep medications such as restoril and trazodone have been shown to be effective in either improving sleep latency or in the maintenance of sleep (Passarella, \& Duong, 2008), sleep medications should be used with a lot of caution. Certain sleep drugs have side effects that may interfere with one's optimal functioning during the day. As such, non-pharmacological approaches to the treatment of insomnia should also be considered.

\subsection{Non-Pharmacological Approaches}

Non-pharmacological approaches are recommended as the initial method to insomnia treatment. In some cases however, pharmacological treatment may be necessary for immediate symptom relief (Passarella, \& Duong, 2008). Non-pharmacological interventions include sleep-hygiene education, stimulus-control therapy, relaxation therapy, and cognitive behavioral therapy (CBT). Other non-pharmacological treatment modalities that have been 
evaluated for the treatment of insomnia include yoga, light therapy, and acupuncture, though these treatments have not been adequately studied (National Institute of Health, 2005).

Sleep hygiene refers to an individual's sleep habits and routines (Shub, Darvishi, \& Kunik, 2009). Education on sleep hygiene focuses on environmental factors and attitudes that negatively affect sleep. Sleep-hygiene recommendations include maintaining a regular sleep schedule, exercising regularly but avoiding exercise too close to bedtime, and avoiding stimulants like caffeine or nicotine right before bedtime (Silber, 2005). Other recommendations include ensuring a comfortable sleep environment such as eliminating noise, decreasing light, and maintaining a comfortable room temperature (Silber, 2005). Evidence indicates that sleep-hygiene education is very effective in treating insomnia in some patients (Shub, Darvishi, \& Kunik, 2009).

Stimulus-control therapy focuses on eliminating maladaptive behaviors, with the overall goal of associating the bedroom with sleep. Patients are instructed to go to bed only when tired, use the bedroom only for sleeping, establish a normal sleep- wake schedule, and avoid daytime napping (Silber, 2005). Patients are also trained to leave the bedroom if unable to fall asleep within fifteen minutes and to return to bed only when tired. Stimulus-control therapy has been shown to be effective in reducing sleep disturbance (Silber, 2005).

Relaxation therapy includes progressive muscle relaxation, biofeedback, and meditation. Relaxation techniques may be especially helpful to patients for whom hyperarousal is suspected as the cause of insomnia symptoms. Relaxation therapy has been shown to improve both sleep latency time and sleep maintenance (Silber, 2005).

CBT addresses patients' anxiety-provoking and erroneous beliefs about sleep. CBT interventions are based on the notion that distorted thoughts about sleep and learned behavior patterns hyperarouse the central nervous system and deregulate sleep cycles, resulting in persistent insomnia. CBT interventions combine empirically tested behavioral, cognitive, and educational procedures to alter faulty beliefs and attitudes, modify sleep habits, and regulate sleep-wake schedules (Whitworth, \& Crownover, 2007). The efficacy of CBT has been well documented and it is recognized as an appropriate treatment for many people with insomnia (Whitworth, \& Crownover, 2007, Zammit, 2007).

\section{Counter Argument}

There are researchers who argue that insomnia is not an impairment unless it interferes with ones social or occupational functioning (Drake et al., 2003). They contend that the amount of sleep required by individuals is very person specific. While many adults need about eight hours of sleep for optimal functioning, many also function effectively with far less than eight hours of sleep. For instance, certain individuals may function effectively with an average of five hours of sleep.

Other researchers argue that insomnia cannot be treated pharmacologically because insomnia rebounds once individuals stop taking medication. Medication thus can only be used to manage insomnia rather than to treat insomnia (Whitworth, \& Crownover, 2007).

\section{Critical Analysis and Limitations}

There are several methodological issues that need to be considered when analyzing any studies that pertain to treatment of insomnia. One area of concern is that a number of the studies under consideration in this paper were conceptual papers (Passarella, \& Duong, 
2008; Whitworth, \& Crownover, 2007; Shub, Darvishi, \& Kunik, 2009) rather than experimental studies. Experimental studies requires (1) random assignment of research participants to experimental and control groups, (2) manipulation of the independent variables in the experimental group but not in the control group, and (3) "comparing the amount of experimental and control group change on the dependent variable" (Rubin \& Babbie, 2001, p 303). Experimental studies are thus preferable in analyzing the efficacy of the various treatment approaches of insomnia.

Another concern is that a number of the studies referenced in this paper were meta-analysis studies that were based on studies with small non-probability samples (Drake et al., 2003; Zammit, 2007). The use of small non-probability samples limits the external validity of the studies and thus their findings cannot be generalized to the wider population. Furthermore, many studies utilized sample subjects who attended sleep clinics. It is possible that many individuals with insomnia do not attend sleep clinics thus the samples utilized may have been biased.

Additionally, a number of the studies referenced in the meta-analysis papers used surveys as the primary means of data collection. Limitations of using survey as a data collection method are many. The major ones include inaccurate recall, differential interpretation of questions, and intended and unintended response error. Also, the reliance of self-report data raises a question of reliability. Specifically, social desirability may be an issue, especially in the case of face-to-face interviews (Rubin \& Babbie, 2001).

Another methodological issue of concern is that some of the studies under consideration were cross-sectional studies. An inherent problem with studies that utilize this design is that they typically aim to understand efficacy processes that occur overtime, yet their conclusions are based on observations made at only one time (Rubin \& Babbie, 2001). An alternative to cross-sectional study is the longitudinal study. Longitudinal designs however are time consuming and affected by dropout.

\section{Social Work Implications}

Insomnia affects millions of Americans. It is therefore very likely that social workers in the mental health field will encounter clients who need help with sleep problems. Social workers thus need to be knowledgeable about the various causes of insomnia and the various treatment approaches that have been shown to be effective in treating insomnia. It is therefore of paramount importance that social workers stay current with the professional literature, and especially on empirical literature that reports on evidence based intervention methods.

Additionally, there is need for social workers to develop effective prevention programs. For example, based on the empirical evidence that shows that poor sleep habits can cause insomnia, social workers can develop educational programs to teach clients healthier sleep habits. These may include teaching clients to avoid daytime naps and heavy meals close to bedtimes. Social workers could also educate clients on the need to have consistent sleep routines. Studies have shown that individuals who avoid daytime naps and have a consistent sleep routine enjoy a much healthier quality of sleep (Edinger, Wohlgemuth, Radtke, Marsh, \& Quillian, 2001). 


\section{$\Lambda$ Macrothink}

\section{Reference:}

American Psychiatric Association (2000). Diagnostic and statistical manual of mental disorders, $4^{\text {th }}$ ed. American Psychiatric Association, Washington DC.

Centers for Disease Control and Prevention (CDC). (2013). Insufficient sleep is a public health epidemic. Retrieved from http://www.cdc.gov/features/dssleep/index. html\#References

Drake, C., Roehrs, T., \& Roth, T. (2003). Insomnia causes, consequences, and therapeutics: An overview. Depression \& anxiety, 18(4), 163-176.

Estivill, E., Bové, A., García-Borreguero, D., Gibert, J., Paniagua, J., Pin, G., Puertus, F., Cilveti, R. (2003). Consensus on drug treatment, definition and diagnosis for insomnia. Clinical drug investigation, 23(6), 351-385.

Edinger, J.D., Wohlgemuth, W.K., Radtke, R.A., Marsh, G.R., Quillian, R.E. (2001). Cognitive behavioral therapy for treatment of chronic primary insomnia: A randomized controlled trial. Journal of the American medical association, 285(14), 1856-1864.

Holcomb, S. (2007). Putting insomnia to rest. Nurse practitioner, 32(4), 28-34.

Lopez, M, A. (2013). Older Adults and Insomnia Resource Guide. Retrieved from https://www.apa.org/pi/aging/resources/guides/insomnia.aspx

Monti, J. (2004). Primary and secondary insomnia: Prevalence, causes and current therapeutics. Current medicinal chemistry - central nervous system agents, 4(2), 119-137.

National Institute of Health. (2005). NIH State of the Science Conference Statement on Manifestations and Management of Chronic Insomnia in Adults. Journal of Clinical Sleep Medicine, 1(4):412-21.

Passarella, S., \& Duong, M. (2008). Diagnosis and treatment of insomnia. American Journal of Health-System Pharmacy, 65(10), 927-934.

Rubin, A. \& Babbie, E. (2001). Research methods for social work $\left(4^{\text {th }}\right.$ ed.). Belmont, CA: Wadsworth.

Sateia, M., \& Nowell, P. (2004). Insomnia. Lancet, 364(9449), 1959-1973.

Shub, D., Darvishi, R., \& Kunik, M. (2009). Non-pharmacologic treatment of insomnia in persons with dementia. Geriatrics, 64(2), 22-26.

Silber, M. H. (2005). Chronic insomnia. New England Journal of Medicine, 353(8), 803-810.

Winkelman J, \& Pies R. (2005). Current patterns and future directions in the treatment of insomnia. Annals of Clinical Psychiatry, 17(1):31-40.

Whitworth, J., \& Crownover, B. (2007). Which nondrug alternatives can help with insomnia?. Journal of Family Practice, 56(10), 836-840.

Zammit, G. (2007). The prevalence, morbidities, and treatments of insomnia. CNS \& Neurological Disorders - Drug Targets, 6(1), 3-16. 